\title{
BABOON MENSTRUAL CYCLES AFFECTED BY SOCIAL ENVIRONMENT
}

\author{
THELMA E. ROWELL* \\ Department of Zoology, Makerere College, \\ University of East Africa
}

(Received 27th November 1968)

\begin{abstract}
Summary. The variation in the follicular phase of the menstrual cycles of seven parous female baboons was studied. The preliminary part of the follicular phase, before oestrus, was longer under conditions of psychosocial stress. Perineal swelling was increased when the normally groupliving female was placed in tactile isolation, and the colour of the swelling changed from bright pink to a pale grey-pink. The latter change was permanently reversed on return to the group. The former was temporarily reversed, but swellings continued to be abnormally large after a long succession of menstrual cycles in which pregnancy was prevented. There was some evidence that the swellings return to normal, after a pregnancy and lactation interval. The implications of these findings are discussed.
\end{abstract}

\section{INTRODUCTION}

Menstrual cycles in primate populations show a characteristic pattern of variation in length. Studies on several species have found a skewed distribution with a specific modal length between 4 and 6 weeks with perhaps three-quarters of all records falling within 5 days either side of the mode, and most of the remainder forming a 'tail' of unusually long cycles (Hartman (1932) on Macaca mulatta; Kuehn, Jensen \& Morrill (1965) on M. nemestrina; Fujiwara, Uchino, Honjo, Imaizumi \& Imamichi (1967) on $M$. irus; Zuckerman (1937) on $M$. nemestrina and Papio hamadryas; Gillman \& Gilbert (1946) on P. ursinus; Yerkes \& Elder (1936) on the chimpanzee).

Because of their perineal swellings, baboons are particularly good subjects for study of the different phases of the menstrual cycle. Zuckerman \& Parkes (1932) were able to show that most of the variation in cycle length occurred in the follicular phase of the cycle, while the luteal phase was remarkably constant in length. This was confirmed by Gillman \& Gilbert (1946) and in the present study. This paper is concerned only with changes in the variable swelling phase of the cycle (equivalent to the follicular phase plus 1 or 2 days), and the luteal phase is not discussed further.

Factors controlling the length of the cycle in chimpanzees were discussed by Young \& Yerkes (1943), who found age and reproductive experience, season,

* Present address: Department of Zoology, Berkeley, University of California. 
gastric illness and individual characteristics to be important variables. They could not find any effect of social environment, but their investigation of this point was not very detailed. Gillman \& Gilbert (1946) noted that baboons became acyclic on an inadequate diet, and under the stress of capture and transport they also noted that it was impossible to draw a line between being acyclic and undergoing an extremely long cycle.

Gillman \& Gilbert (1946) demonstrated that the size of the baboon perineal swelling is very precisely related to blood concentrations of oestrogens and progesterone, but swelling size may also be altered by external factors: DeVore (1965) mentions a rapid decrease in swelling size of wild females that became involved in fights, and forcible handling of caged baboons sometimes has the same effect. The following case also suggested the possibility that social environment could affect swelling size: two females were captured with newborn infants and kept in very small cages with these infants for a year. Baboons normally begin to cycle again, showing full swellings, when the infant is about 6 months old, or within 2 or 3 weeks of a younger infant being removed. When these females came to the author's colony, 4 months after their infants had been removed, they were menstruating but showing barely detectable fluctuations in perineal skin oedema. Two months later they were caged together and within a week both had begun to develop normal swellings which reached the size later found typical for each. This seems comparable to Gillman \& Gilbert's observation that females which had become acyclic under the stress of capture would begin to cycle at once if caged with "one of the old inmates of the colony". Both the causes and effects of the above examples were extreme, and the following experiment was planned to see if simple isolation of normally group-living females would have an effect on some obvious characters of the menstrual cycle: size and colour of the swelling and length of the swelling phase. In the event, comparison of group and isolated environments did not prove to be the most interesting way of classifying the data.

\section{ANIMALS AND METHODS}

Seven parous females, from a breeding group of baboons (Papio anubis) whose menstrual cycles had been recorded for the previous 4 years, were used; two other females remained in the group throughout the experiment, undergoing normal reproductive life. The main group lived in a run with a total floor area of 600 sq. $\mathrm{ft}$. The females were taken from the group at the beginning of a menstrual cycle and placed in small pens $(5 \times 12 \times 6 \mathrm{ft})$ from which they could see and hear but not touch at least two other females. Each stayed in an 'isolation' pen until she had completed that cycle and four (in two cases, five) more cycles, and was then returned to the group during the menstruation of the next cycle. For this and four further cycles, pregnancy was prevented by separating the females from males for the probable fertile period: they were confined to a $120 \mathrm{sq}$. $\mathrm{ft}$ section of the living run with other females, separated from the males by 1 in. mesh. Despite these precautions, one female became pregnant on return to the group and was dropped from the series. After five cycles, the females were 
allowed to stay with the males, and their cycles were recorded until they became pregnant.

The state of the perineal skin was recorded daily. The size of the swelling was estimated on a ten-point subjective scale; photographs were less reliable, and used only in confirming impressions of changes in maximum swelling size over a long period. Direct measurement was not possible because the animals were not tame to handling. Colour of swellings was noted, but no quantitative way of recording colour in the differing light conditions was devised.

From the pre-isolation period, all cycles were used for which there were complete records from menstruation to subsidence of the perineal swelling while the female was in the group, i.e. the first swelling after a lactation interval, not being preceded by menstruation, was omitted, but swellings during which conception occurred, if preceded by menstruation, were included. The small number of cycles recorded for each female over 4 years is due to the high conception rate. Although one female swelled as many as seven times before conceiving, only seven of seventeen conceptions that went to term were preceded by more than one menstruation. (The birth interval in a wild population was also around 14 months.)

\section{RESULTS}

The swelling phase: general

Lengths of all swelling phases recorded for the seven females are shown in Text-fig. 1. They have the expected skewed-normal distribution, with a peak of

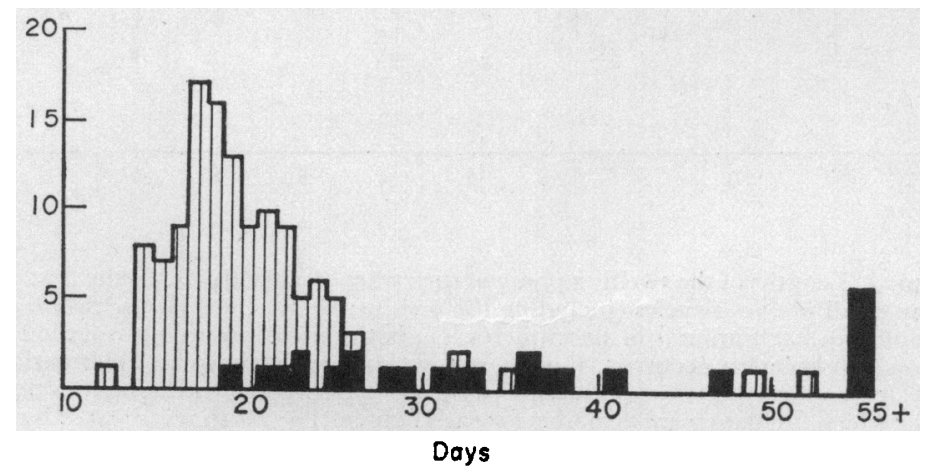

TExT-FIG. 1. Lengths of the swelling phases of all cycles recorded from the seven experimental females. In black, cycles in which the female was known to have been beaten, or was moved in or out of the group around the time of the initial menstruation.

frequency at 17 days. Individuals had their own characteristic range of variation, e.g. the shortest swelling recorded for Emma was 14 days and for Collar, 21 days. Nevertheless, $70 \%$ of all swellings were within 5 days of the most frequent length, while the remaining twenty-seven cycles were all unusually long in the swelling phase.

When females were moved, either in or out of the group, at menstruation, the succeeding swelling phase was often longer than usual (Text-fig. 2). On return 
to the group, the females were all attacked by the resident females to some extent, which suggested a possible relation between cycle length and aggression. A distinction had to be made between sudden brief attacks, often made on lower-ranking females at or near full swelling, which might cause severe wounds but did not appear to cow the recipient and did not affect her menstrual cycle, and long-drawn-out beating-up accompanied by repeated bouts of threats, which produced abject behaviour in the recipient. When these latter occurred immediately before, during or just after menstruation, the succeeding

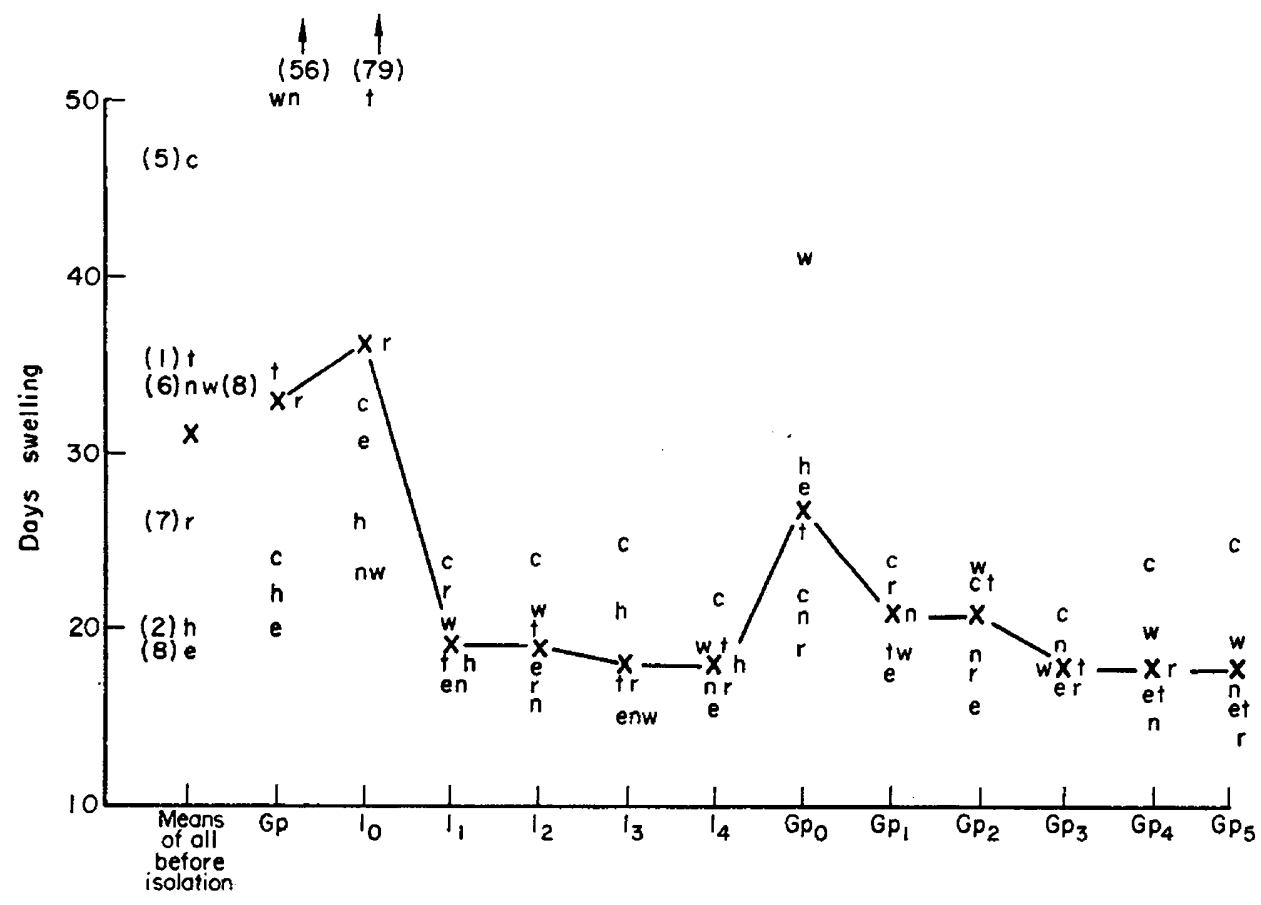

TEXT-FIG. 2. Lengths of the swelling phase of the cycles of individuals. In the first column, the mean of all previous cycles (including the one just before isolation) with the number of examples for each animal in parentheses. $G_{p}$ is the cycle preceding isolation, $I_{0}$ the cycle in which isolation occurred. $I_{1}$ to $I_{4}$ are cycles completely in tactile isolation, $G p_{0}$ is the cycle in which the female returned to the group. $G p_{1}$ to $G p_{5}$ the next cycles in the group for which full data were available for each female. The mean is indicated by crosses.

swelling phase tended to be longer. Though assessment of the severity of the effect could only be subjective, there was possibly a rough positive correlation between severity and effect on duration of swelling phase. Female Collar was first released into the group with a large swelling: she was beaten by the resident females and her swelling began to subside. Menstruation followed after her typical luteal interval, giving the shortest recorded swelling phase of only 12 days (not included in the sample because it did not occur in the group). This was the only occasion on which beating started during full swelling-probably because the male would have intervened on behalf of a familiar consort-and the only occasion on which beating was associated with a shortened swelling phase: 
for want of further evidence, the two events are assumed to be related. Beating continued and Collar's next cycle was one of the longest recorded, with a swelling phase of over 100 days.

In Text-fig. 1, swelling phases in which the female was moved or beaten are shown in black. It is obvious that they tend to be longer than usual: only $3 \%$ of those which fall within 5 days of the most frequent length had a history of moving, while $59 \%$ of those outside that range had a history of moving or beating, including all but two of those longer than 35 days. The records for the remaining two are incomplete, and are from an animal that may well have been beaten at that time.

In the extremely long swelling phases included here, and from three other instances in females outside the study, the perineal skin remained flat after menstruation (typically, swelling starts on the 2nd or 3rd day of menstruation) for an interval of up to 3 months. The baboons of Gillman \& Gilbert which became acyclic also did so at this point of their cycles.

\section{The swelling phase: effect of tactile isolation}

Lengths of swelling phase for individual females before, during and after isolation are shown in Text-fig. 2. The average length of swelling phases before isolation is high because the sample includes the exceptionally long cycles which occurred when some animals were moved into the group. Five of the seven showed an increase on isolation: the other two had just had unusually long cycles, so their first swellings in isolation were actually shorter than in these preceding cycles. Swelling phases in complete isolation were all short and varied very little. On return to the group, all but one had a longer swelling phase, but for five females this was shorter than the last cycle before isolation. Thereafter, the mean length decreased until in the third cycle completely in the group, the low level first seen in isolation was reached and then maintained. Allowing the females to stay with the males $\left(\mathrm{Gp}_{5}\right.$ and later) did not affect swelling-phase length.

\section{'Preliminary' and 'oestrous' phases}

Baboons do not begin to mate or increase their interactions with adult males until their swellings become fairly large. Oestrous behaviour appears quite suddenly in the cycle, often accompanied by brightening of the colour of the swelling, and at a size of swelling which is typical of the individual. It is thus possible to divide the swelling phase of the cycle into a preliminary phase, from start of menstruation to start of oestrus, and an oestrous phase which continues until subsidence. In isolation, behavioural criteria were not available so the correlated swelling size was used as the criterion for the oestrous phase throughout.

In Text-figs. 3 and 4, the preliminary and oestrous phase lengths are plotted separately. The preliminary phase showed the same changes throughout the experiment as did the swelling phase as a whole (Text-fig. 2), while the oestrous phase remained almost constant, though after isolation the average length was slightly shorter than before. 
Size and colour of swellings

Changes in swelling size are illustrated in Text-fig. 5. Although no two animals showed exactly the same pattern, the majority (at least five of the seven) changed in the same way at each stage. Swellings became bigger on isolation

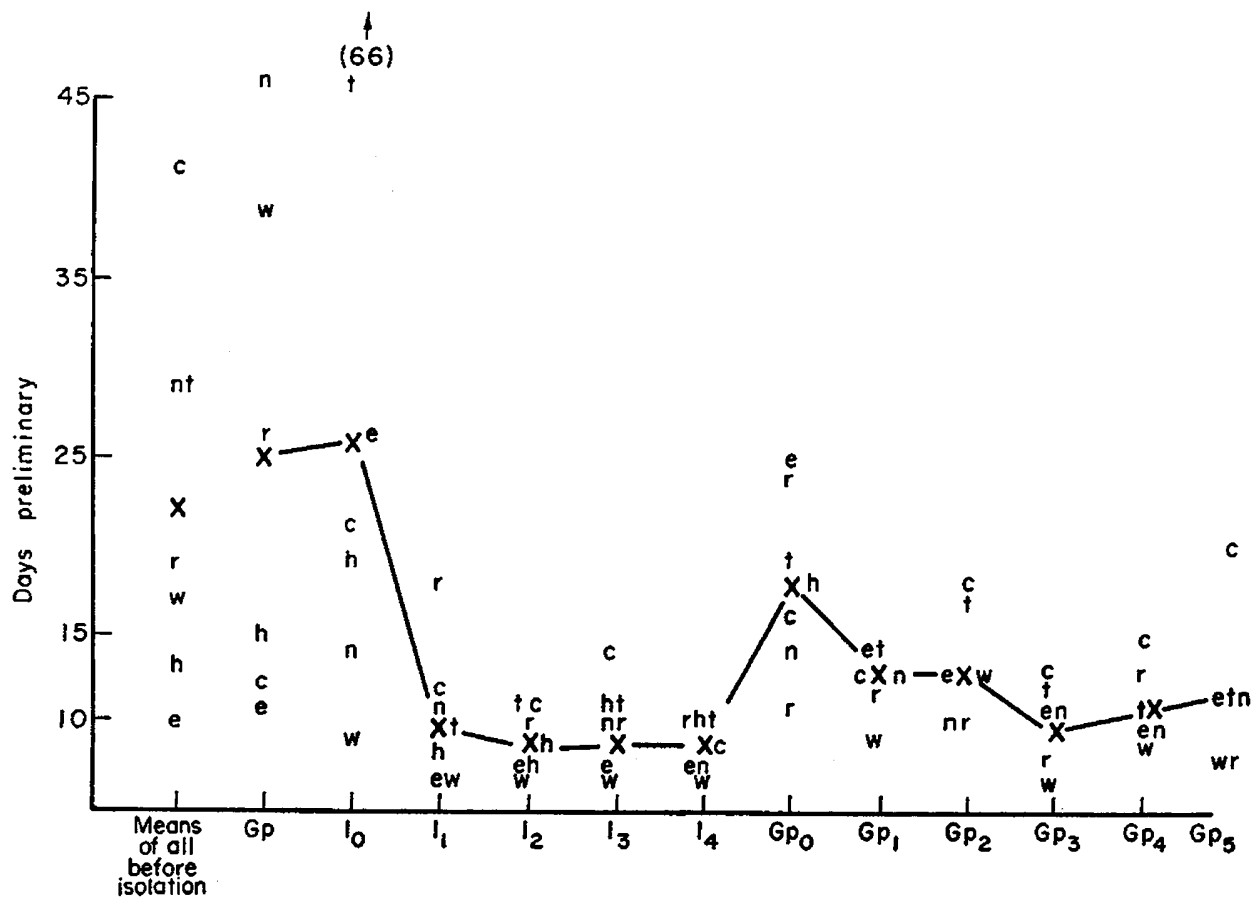

TEXT-FIG. 3. Lengths of the preliminary part of the swelling phase in individuals. Conventions as in Text-fig. 2.

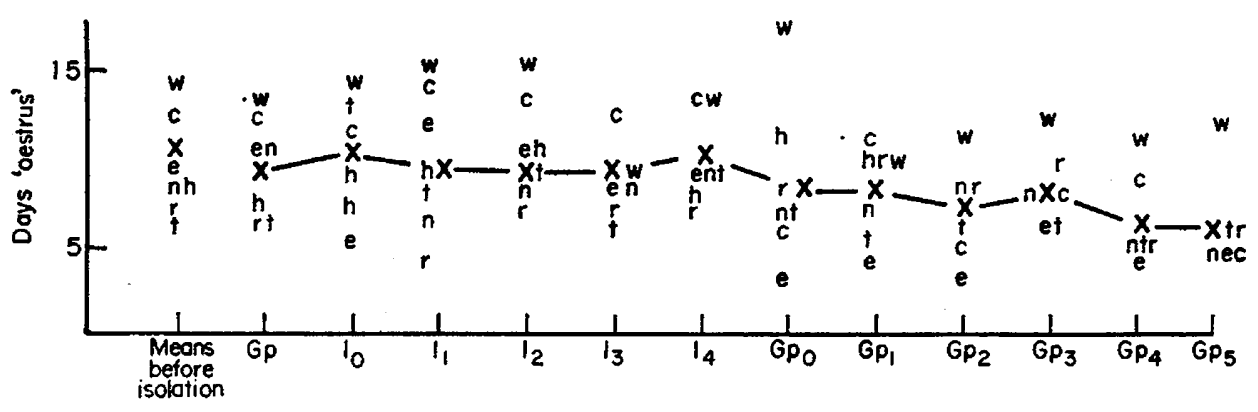

TEXT-FIG. 4. Lengths of the oestrous part of the swelling phase of individuals. Conventions as in Text-fig. 2.

and decreased on return to the group. However, post-isolation swellings were still noticeably larger than before isolation: before, most swellings were within the range of size typical of wild baboons; afterwards, they were larger than was normally seen in the wild. The swellings of females not involved in the isolation experiment did not increase in size over the same period, (these females had 
been pregnant in the interval). Similarly, the female, which became pregnant on return to the group, did not produce very large swellings when she began to cycle again after a stillbirth, but returned to the small swellings typical of her pre-isolation period (Text-fig. $4, \mathrm{f}$ to $\mathrm{h}$ ). The history of the female, Ruth, is also relevant: nulliparous and having menstruated regularly for 3 or 4 years, her swellings when she joined the group were enormous. The oedema even extended into the lateral naked skin which is not normally involved in the swelling.

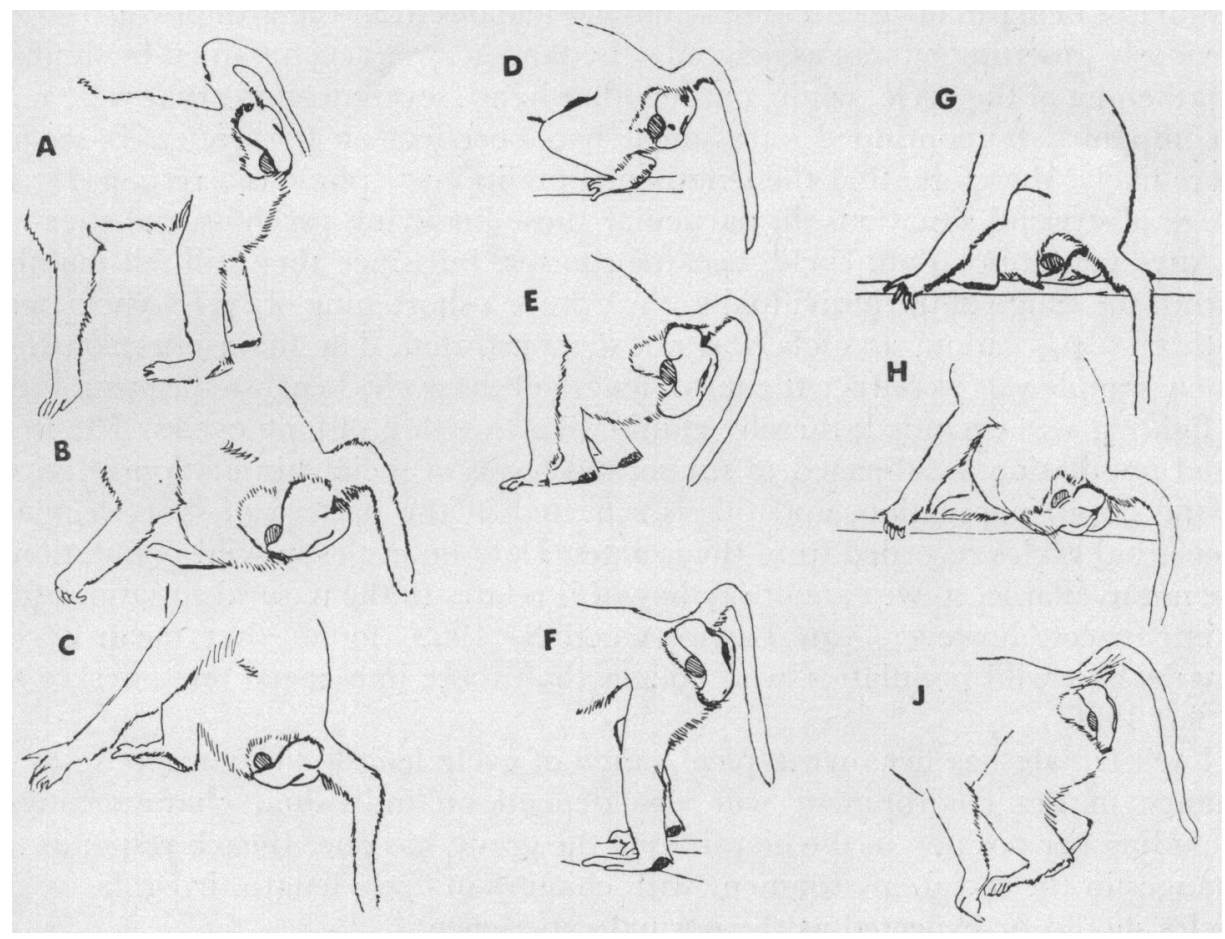

TEXT-FIG. 5. Changes in maximum swelling size (tracings from photographs). A to $\mathrm{C}$ and $\mathrm{D}$ to $\mathrm{F}$ : two females at maximum swelling before, during and after tactile isolation. $\mathbf{H}$ and $J$, female Harriet during and after isolation; $G$, the same female when cycles were re-established after still birth, to show that her swelling had returned to the small size typical of pre-experimental cycles.

Between the births of her infants, very small swellings developed, and she then entered the present experiment. After the year of successive cycles which this involved, she was again producing huge swellings with lateral extension. (Swellings in this group of baboons, however, have never approached the sizes noted by Gillman \& Gilbert (1946) or recorded by Zuckerman (1937) as being 10 or $15 \%$ of the body weight.)

Like the shape and size, the colour of the swelling is also an individual characteristic. In the wild or in the group, this was usually a shade of bright pink. In isolation, the large swellings were pale, grey-pink shades; on return to the group, each animal again became bright pink in oestrus as she had before isolation. 


\section{DISCUSSION AND CONCLUSIONS}

The swelling-phase length in this group of baboons did not change with the season, unlike those of chimpanzees (Young \& Yerkes, 1943). All factors which appeared to cause variation in the swelling phase were changes in the social aspect of the environment. When females were beaten-up by other females, their menstrual cycles were lengthened: specifically, the interval between menstruation and the onset of oestrus was increased. This cannot be attributed to the physical trauma of being mauled, because removing females from the group, which was obviously upsetting but not associated with damage, was accompanied by similar lengthening of the cycle, while, on the other hand, severe wounds that were not accompanied by continued harassment had no effect on the cycles. It seems more likely, therefore, that the lengthened preliminary phase is a response to a range of stressful situations, in particular those involving psycho-social stress.

After tactile isolation, cycles became shorter, but since they still fell mainly within the range of the individual in the group, a shortening of cycles associated with tactile isolation, as such, was not demonstrated. The main effect on the whole sample was to reduce the mean length of the cycles because the possibility of fighting was excluded, thereby eliminating the 'tail' of long cycles. The isolated population is subjected to subnormal levels of social stimulation relative to the group population, and this is reflected in the pattern of short regular menstrual cycles recorded from the isolates. Data on cycles in wild populations are not available, so we cannot say how this relates to the natural situation; for chimpanzees, however, van Lawick-Goodall (1969) found that mean cycle lengths in a wild population were longer than those in a caged one (Yerkes \& Elder, 1936).

Each female has her own typical range of cycle lengths. Her response to a change in her environment will also depend on individual characteristics, including her position in the hierarchy if the group has one. If each responds to changes in the social environment with changes in cycle length, irregularity of cycles should be expected as the normal experience.

The maximum size of swellings increased as soon as females were in tactile isolation and on return to the group they became smaller, but later increased again to sizes intermediate between those of isolation and those seen before the experiment. It is suggested that two factors worked together here, both isolation, and the experience of repeated cycling itself, producing larger swellings. Gillman \& Gilbert (1946) noted increase in swelling size in successive cycles after lactation, and also after puberty, describing it as a process of maturation. But since Papio anubis females regularly conceive in the swelling after the first menstruation following puberty or a lactation interval, the 'immaturity' of these swellings is surely a matter of subjective judgement. On the other hand, the enormous swellings of captive baboons, which have cycled for several years without a pregnancy and lactation interval, might well be regarded as pathological. The perineal skin is not adapted to repeated periods of oedema at short intervals, but to occasional swelling with long rest-periods of about a year between bouts of two or three oedematous episodes.

An isolated baboon is relatively inactive, whereas an oestrous baboon in a 
group is characterized by her restlessness. The striking loss of colour in swellings in tactile isolation could be caused by a reduction of peripheral bloodflow in the inactivity of isolation, and it could also be indicative of other physiological differences associated with this striking change in behaviour in the two situations.

For research on reproductive problems, long series of short regular menstrual cycles might be convenient and these can be most reliably obtained by keeping a baboon in tactile isolation. It is clear, however, that continuous cycles are not the condition for which baboon physiological mechanisms have evolved, and differences in the size, appearance and duration of the swelling in normal (i.e. wild) and isolation situations could be only the more obvious signs of more fundamental differences in the physiology of cycles. The effect of tactile isolation on a social primate is to produce an environmental deficit of severity comparable to, say, a mild dietary deficit and results obtained in such circumstances should be evaluated accordingly.

\section{AGKNOWLEDGMENTS}

This research was financed by the Medical Research Council of Great Britain. Mr S. Bayoha made some of the observations on which the results are based. Dr M. G. Pike gave advice on statistical matters.

\section{REFERENCES}

DeVore, I. (1965) Male dominance and mating behaviour in baboons. In: Sex and Behaviour. Ed. F. A. Beach. Wiley, New York.

Fujiwara, T., Uchino, I., Honjo, S., Imaizumi, K. \& Imamichi, T. (1967) Normal range of the menstrual cycle of Cynomolgus monkeys under laboratory conditions. Fap. F. med. Sci. Biol. 20, 505.

Gillman, J. \& Grlbert, G. (1946) The reproductive cycle of the Chacma baboon (Papio ursinus) with special reference to the problems of menstrual irregularity as assessed by the behaviour of the sex skin. S. Afr. F. med. Sci., 11, 1. Biol. Suppl.

Hartman, C. G. (1932) Studies in the reproduction of the monkey, Macacus (Pithecus) rhesus, with special reference to menstruation and pregnancy. Contr. Embryol. 23, 1.

Kuehn, R. E., Jensen, G. \& Morrill, R. K. (1965) Breeding Macaca nemestrina: a problem in birth engineering. Folia Primatol. 3, 251.

van Lawrok-Goodall, J. (1969) Some aspects of reproductive behaviour in a group of wild chimpanzees, Pan troglodytes schweinfurthi, at the Gombe Stream Chimpanzee Reserve, Tanzania, East Africa. F. Reprod. Fert. Suppl. 6, 353.

Yerkes, R. M. \& Elder, J. H. (1936) The sexual cycle of the chimpanzee. Anat. Rec. 67, 119.

Young, W. C. \& YeRkes, R. M. (1943) Factors influencing the reproductive cycle of the chimpanzee, the period of adolescent sterility, and related problems. Endocrinology, 33, 121.

Zuckerman, S. (1937) The duration and phases of the menstrual cycle in primates. Proc. zool. Soc. Lond. 107, 315.

Zuckerman, S. \& Parkes, A. S. (1932) The menstrual cycle of the primates, pt. IV. The cycle of the baboon. Proc. zool. Soc. Lond. 102, 139. 